\title{
Review
}

\section{Vaccines for tumor prevention: a pipe dream?}

\author{
Guido Forni
}

Accademia Nazionale dei Lincei, Rome, Italy

\begin{abstract}
Whether or not a tumor expresses peculiar antigens that differentiate it from normal cells was intensively investigated in the 1950s. A conclusive answer was provided in 1960 when George Klein showed that a tumor can be rejected by the immune response elicited by a vaccine administered to the same mouse in which the tumor was induced. Whether immunogenicity was a feature restricted only to tumors artificially induced by viruses or by high doses of chemical carcinogens was then hotly debated until Terry Boon showed, in the 1980s, that almost any tumor can be recognized by a syngeneic immune system triggered by an appropriate cancer vaccine. However, the therapeutic efficacy of vaccine-induced immunity against an advanced tumor is marginal. The combination of an anti-tumor vaccine with new sophisticated maneuvers to contrast tumor-induced suppression may yield new and effective therapeutic strategies. Also, the exploitation of tumor vaccines to prevent tumors in cohorts of people with a specific risk of cancer may become a fresh strategy with great potential to control tumor onset.
\end{abstract}

Key words: vaccine; tumor; tumor antigens; oncoantigens; T cells; antibodies; tumor prevention.

J Infect Dev Ctries 2015; 9(6):600-608. doi:10.3855/jidc.7201

(Received 26 April 2015 - Accepted 27 May 2015)

Copyright () 2015 Forni This is an open-access article distributed under the Creative Commons Attribution License, which permits unrestricted use, distribution, and reproduction in any medium, provided the original work is properly cited.

\section{Introduction}

The perception that immunity may be exploited to control tumor growth has existed for almost one hundred years. As soon as the ability of vaccines to elicit an immune memory able to overpower microbial infections was recognized, several attempts were made to apply vaccination to protect against tumors. Cancer (Latin for "crab") was thought to be a parasite against which an immune reaction could be elicited. When the first mouse-transplantable tumors were available, several experiments were done, immunizing mice with variously modified and inactivated tumor cells. The growth of the engrafted tumor in control mice and its rejection by immunized mice suggested the possibility of eliciting a protective immunity against cancer. However, in several cases, tumor rejection was due to a boosted response against not-yet-defined histocompatibility antigens expressed on the tumor cell surface, and not against an abnormal molecule connected to the neoplastic state of the cell (the socalled tumor associate antigen, or TAA) [1]. For a long time, it was difficult to prove that tumor rejection was not due to a vaccine-boosted reaction against some residual heterozygosity. Even when wellcharacterized inbred strains of mice were available, the suspicion that unnoticed residual histocompatibility within mice of an inbred strain was the cause of tumor rejection by immunized mice was almost unavoidable [2].

The formal proof of the possibility of using a vaccine to immunize against tumors was provided by Klein et al. in 1960 [3]. In this study, sarcomas were induced by methylcholanthrene in 16 inbred mice. As soon as the tumors reached approximately one centimeter in diameter, each tumor was surgically removed. Each surgically treated mouse was then repeatedly immunized with heavily irradiated cells of its own sarcoma maintained through passages in other inbred mice. When the surgically treated and immunized mice were challenged with a lethal dose of their own sarcoma cells, a significant resistance to tumor growth was observed in 12 out of 16 mice. As the authors proudly claimed, these results showed, beyond doubt, that a vaccine can afford a significant protection to a mouse against its own surgically removed tumor.

The demonstration of the possibility of eliciting a protective immune response against a tumor along with the progressive availability of precisely standardized strains of inbred mice spurred numerous investigators around the world to study the features of vaccine-elicited anti-tumor reactions. In the great majority of these studies, groups of inbred rodents were variously immunized against a syngeneic tumor 
and then challenged with a lethal dose of cells of the same tumor injected subcutaneously, intramuscularly, or orthotopically [4]. These immunization-challenge experiments are easy to set up and standardize, and quickly provide an assessment of vaccine efficacy.

In the 10-15 years that followed the pioneering Klein's paper, overwhelming experimental evidence stemming from innumerous immunization-challenge experiments was showing that protective immune memory elicited by an anti-cancer vaccine against a syngeneic tumor engrafted in an immunized rodent rests on T-cell reactivity [5,6], while antibody [7] and $\mathrm{B}$ cells [8] have no role or even may interfere with the efficiency of the memory rejection.

While these data provide a strong rationale for cancer immunotherapy, all the clinical attempts that were made to exploit a vaccine in cancer therapy provided marginal results. These discouraging clinical observations spurred a radical criticism of the significance of data on tumor immunogenicity obtained in the immunization-challenge experiments. It was argued by Hewitt that immunotherapy of cancer, as well as the theory of cancer immunogenicity on which it rests, was based on data that had been obtained from transplanted tumors which entailed artifactual immunity associated with their viral or chemical induction [9]. These tumors should not be considered acceptable as valid models of clinical cancer. In fact, when inbred mice from Hewitt's laboratory were immunized with lethally irradiated cells of syngeneic tumors of spontaneous origin, no increased resistance to a subsequent tumor engraftment was ever observed. This observation was true with seven distinct spontaneous tumors. The conclusions of Hewitt and colleagues were manifestly negative: "We suspect that the almost exclusive resort of tumor immunologists to chemically induced, virusinduced or tumors is calculated to eke out evidence supporting the general theory of effective tumor immunogenicity which otherwise might fail to attract the very large attention it receives. We venture to suggest that expansion of an already vast volume of literature would be considerably restrained if publication of papers drawing clinical implications from animal experiments in this field were made conditional upon the use of tumor systems free from the more obvious stigmata of artefactual immunity" [10].

What was the rebuttal of Hewitt's British skepticism? It was claimed that the use of artificially highly immunogenic tumors was required in order to model a complex problem in a simpler way.
Admittedly, the majority of tumors exploited in the immunization-challenge experiments were artificially immunogenic, but their immunogenicity was instrumental for evaluating how an effective immune memory could be elicited against a tumor. These tumors are not the real thing, but are rather models to enable better study of real tumors [11,12].

While a skeptical vision of tumor immunogenicity was spreading around, the use of more sophisticated vaccination techniques showed that even tumors that, in more conventional experiments, were unable to induce a protective immune response were promptly rejected when syngeneic recipient mice were preimmunized with vaccines able to trigger an appropriate response [13]. A systematic work by Boon in Belgium showed that by selecting or inducing variants of non-immunogenic tumors, it was possible to elicit a protective immune memory against parental tumors otherwise classed as non-immunogenic [14].

These studies helped to shift the problem of the apparent non-immunogenicity of tumors; the issue was to devise more effective vaccines able to elicit an immune reaction against a TAA that otherwise would be ignored by the immune system. Vaccines based on inactivated tumor cells were effective in eliciting an immune memory against very immunogenic tumors. With more realistic tumors, these simple vaccines have to be substituted by more sophisticated new vaccines able to trigger an immune recognition of TAA expressed on the cell membranes of tumors that otherwise would not be recognized by the immune system

As T cells appear to be the central cells (if not the only cells) on which tumor immunity rests, the study of TAA progressed with the definition of what a $T$ cell perceives. The recognition that $\mathrm{T}$ cell antigen receptor (TCR) does not interact with foreign molecules but only binds to self-histocompatibility molecules, presenting antigen peptides in their groove [15], spurred the study of what autologous $\mathrm{T}$ cells recognize on the tumor cell membrane. The front-running $\mathrm{T}$. Boon laboratory showed that melanoma cells from primary human tumors were able to stimulate autologous $\mathrm{T}$ cells to proliferate and become cytotoxic for autologous melanomas. Moreover, clonal analysis of cytotoxic $\mathrm{T}$ cell combined with genetic and biochemical approaches led to the molecular identification of three classes of antigens associated with human tumors. The first class comprises antigens encoded by genes such as MAGE, BAGE, and GAGE, which are expressed in various tumors of different histological origins, but not in normal tissues other 
than testis. The second class represents differentiation antigens encoded by genes that are only expressed by tumors of certain tissues, such as tyrosinase, MelanA/MART-1, gp100, and gp75 in melanoma and normal melanocytes. The third class includes antigens produced by unique point mutations in genes that are ubiquitously expressed [16-17]. These findings opened a completely new way of thinking about how to immunize patients with molecularly defined tumor peptides or with autologous antigen-presenting cells pulsed with the peptide [17].

Was this the end of the search for tumor antigens? Of course not, but certainly these data were of crucial conceptual importance since they showed that distinct groups of molecules anomalously expressed by human tumors may be recognized by the immune system, both spontaneously or in a much more intense and persistent way, following vaccination. As virtually any mutant, overexpressed or abnormally expressed, protein in cancer cells can serve as a target for cancer vaccines, the number of potential cancer antigens is almost limitless. Moreover, even the loss or rarefaction of a normal membrane antigen can be perceived by the immune system and trigger a quick killing of the target cells [18].

Seventy-five TAAs have been prioritized by a pilot project of the USA National Cancer Institute on the basis of criteria weighting (a) their therapeutic function; (b) immunogenicity; (c) their role in antigen in oncogenicity; (d) specificity; (e) expression level and percent of antigen-positive cells; (f) stem cell expression; (g) number of patients with antigenpositive cancers; (h) number of antigenic epitopes; and (i) cellular location of antigen expression. If this prioritization well reflects the current status of the cancer vaccine field, it should clearly be stated that these rankings are dynamic, given that priorities change as knowledge accrues from new studies [19].

Despite the precise molecular identification of TAA, therapeutic vaccination of cancer patients with the TAA of the MAGE family or with TAA differentiation was followed by tumor regressions in only a small minority of the patients. Nevertheless, $\mathrm{T}$ cell responses to the vaccines showed a correlation with tumor regressions [17].

\section{The wretched battle for a cancer vaccine}

The search for cancer-associated antigens has been successful. At present, we know of a large number of molecules anomalously expressed on tumor cells that may be the target of an anti-tumor vaccine, and numerous new molecules are being identified $[19,20]$.
New vaccine technologies allow immune tolerance to be overcome and induce a marked immune reaction against almost every TAA in sophisticated and realistic mouse cancer models [21]. In these models, we also have a large array of experimental data showing the potential activity of these vaccines to inhibit cancer development. So, why are data so far emerging from clinical trials at best marginal, if not negative $[17,22]$ ?

Multiple reasons are at the basis of clinical failure of tumor vaccines. The first one is that in the real world, a vaccine should be able to trigger a marked immune reaction in a patient who, in many cases, is an elderly person who has already received multiple conventional anti-cancer treatments. As in the case of old mice, elderly people often display an impaired ability to produce an effective immune response to a new antigenic stimulation [23]. This impaired immunological reactivity can be worsened by conventional anti-tumor therapies that, in the majority of cases, decrease the reactivity of the immune system. Lastly, several human cancers are endowed with the ability to suppress immune response. Tumors may release suppressor cytokines and display on the cell membrane molecules that block the induction of the immune response. In addition, the increased expression of TAA by proliferating tumor cells the absence of costimulatory stimuli triggers a state of immune anergy specific to that given TAA [24].

Thus, when an anti-cancer vaccine is exploited in tumor therapy, it is asked to accomplish an exceedingly difficult task. The immune response induced has then to deal with a diffuse disease. When an anti-cancer vaccine is employed in cancer therapy, it is asked to induce a strong immune reactivity, an exceedingly difficult outcome. Even when a vaccine is able to induce an immune response in a cancer patient, the effector arm of the immune response is confronted with a diffuse dynamic disease often represented by numerous tumor masses difficult to penetrate for both $\mathrm{T}$ cells and antibodies.

The accumulation of these factors has already made desperate the therapeutic mission of anti-tumor vaccines; however, this is not the whole picture. The purposeless proliferative ability of cancer may even be enhanced by the vaccine-induced immune reaction. As a tumor progresses, genetic instability, one of the hallmarks of cancer, allows the diversification of various clonal sub-populations of tumor cells. An established tumor can be seen as an aggregate of millions of genetically different and unstable cells, each one endowed with an enormous clonogenic 
potential. When vaccine-elicited immune reactivity is not sufficient to destroy the entire tumor, clones that are more susceptible to immune attack are those that are eliminated. This immune selection edits the clonal composition of a tumor by positively selecting tumor clones that less express the target antigen and therefore are better able to withstand the immune response, an event known as immunoediting [25]. Often, less differentiated cells endowed with a more aggressive behavior form the clones able to escape the immune attack. After an initial shrinkage due to the killing of more immunogenic tumor clones, the immune attack may result in a more anaplastic tumor that is capable of expanding with a higher proliferative and metastatic potential $[25,26]$.

Is the emerging scenario suggestive of the fact that there is little future for therapeutic vaccination in cancer? If past and current data seems to suggest so, the clinical cancer situations are so differentiated that, in many cases, a rational exploitation of technologically advanced anti-tumor vaccines is emerging [27]. Vaccine-elicited immunity may become a component of multi-component therapies, since in many cases immune reactions against tumor antigens contribute to the long-term clinical benefits of anti-cancer treatments such as chemotherapy and radiotherapy [19], or may be exploited to control tumor relapses [26].

As the efficacy of vaccines is diminished by the tumor-driven expansion of several immunosuppressive mechanisms, strategies counteracting tumor-induced suppressor mechanisms during vaccination can make the difference between an ineffective vaccine and an effective one. The progressive elucidation of cellular and molecular mechanisms exploited by a tumor in order to suppress the immune response is providing a new and effective way to counteract cancer cell's ability to evade a vaccine-induced immune response. Mouse models clearly show that when it is coupled with the depletion of T-regulatory $\left(\mathrm{T}_{\text {reg }}\right)$ cells, an antitumor vaccine is efficacious at tumor stages at which vaccination alone is ineffective. A long-lasting tumor immunity is induced, and the intensity of the immune reaction is enhanced [28,29]. However, $\mathrm{T}_{\text {reg }}$ cells are not the sole suppressive cell population that expands during tumor progression. Immature myeloid cells form another important immunosuppressive cell population whose expansion goes along with tumor progression [30,31]. Various ways to inhibit immature myeloid cell generation, accumulation, and function are now envisaged [31].
It is also conceivable that the combination of antitumor vaccines with the administration of antibodies that block immune inhibitory pathways switched on $\mathrm{T}$ cells by CTLA-4 and PD-1 membrane receptors following their interaction with ligands expressed on the cell membrane of tumor cells, cancer cells will markedly enhance the effectiveness of the therapeutic activity of anti-cancer vaccines; this is a practice known as checkpoint blockade [32]. A sophisticated interference with tumor-borne regulatory and suppressor mechanisms may be a key issue to endow anti-tumor vaccines with therapeutic efficacy.

\section{The responsibility of experimental models}

Experimental models of the tumor-host relationship are constructed to emphasize a few basic features of a complex and multifaceted situation. Their responsibility is great because the experimental findings they yield influence the rationale for the implementation of therapies based on fresh concepts [21].

Tumor engraftment in immunized syngeneic rodents (mice, rats, guinea pigs) is a neat and quick way to study the basic features of the vaccine-elicited anti-tumor reactivity. Experimental models of this kind have been and still are popular since transplanted tumors usually grow and kill their hosts in a matter of few weeks [5]. This brief time scale allows for the gathering of a large amount of data in a short period of time on the protective potential of a vaccine. However, the brief time scale of these immunization-challenge experiments is also one of the original issues contaminating data emerging from these models. In fact, the short time scale disguises a few critical features that characterize the long-lasting tumor-host relationship, such as the selection of tumor clones that escape the immune attack [33], the progressive anergy acquired by the immune system to the TAAs expressed by tumor cells, the various kinds of tumorborne immunosuppression, and the tumor's ability to shelter itself from immune attack. The error intrinsically inherent in experiments of this type is the exploit of fast mouse models to study a chronic disease. Transplanted tumors usually grow and kill their hosts in a matter of a few weeks and are thus an acute model of a relationship that, in a cancer patient, may last several months or years [21].

A second issue in the immunization-challenge type of experiments rests on the fact that the recipient rodent is immunized first while the tumor is engrafted once a specific immune memory against tumor TAAs is fully realized [5]. The vaccine elicits an anti-tumor 
response in healthy mice whose immune systems are not yet impaired by tumor growth. When the immunized mice are challenged by the tumor, an immediate immune response attacks the just-engrafted tumor cells that did not have the time to make operative the sheltering and escaping mechanisms. The complete eradication of the engrafted tumor cells by pre-existing immunity is a quick occurrence. Indeed, these immunization-challenge experiments highlight the central role of cytotoxic $\mathrm{T}$ lymphocytes ( $\mathrm{T}$ killer cells) in tumor rejection. This is an indisputable acquisition coming from these short-term experiments. Probably, the activity of $\mathrm{T}$ killer cells does not have such an absolute importance in a longlasting tumor-immune reactivity relationship.

What is the responsibility of tumor models? Whether deliberately or unthinkingly, data from immunization-challenge experiments have been exploited as the premise for clinical trials aimed at curing cancer. The inappropriateness of this transposition is illustrated by the fact that when mice are first challenged with a tumor and then immunized, the elicitation of an effective therapeutic response is difficult, and only a minority of mice is cured. Even this limited efficacy is achieved only when the vaccine is administered in the first few days after the tumor challenge [34]. The difficulty in curing transplantable tumors may partially be due to the kinetics of tumor growth that may be so fast to overtake the time necessary for the vaccine to induce an immune response. However, even with slow-growing tumors, most published data on the curing of mouse tumors by vaccination must be classed as discouraging.

Mice that naturally develop a tumor as a consequence of a defined gene alteration artificially inserted in their genome (the so-called genetically engineered mice, GEM) constitute a somewhat more realistic experimental model. A defined genetic predestination to develop a tumor, the slowness of tumor progression through defined pathological stages, the natural occurrence of disseminated cells and metastasis, and the long-lasting interaction between the evolving tumor and the host immune system are the appealing features of cancer-prone GEM models $[21,35]$. However, several features make the breeding of GEM and their exploitation in immunization experiments very demanding. Because of the realistic slowness of tumor progression, experiments to assess the vaccine-induced protection may last for one or two years, a time equivalent to the normal life span of mice [36]. Therefore, the choice of a GEM model of cancer is a delicate compromise between its features, its handiness, and the specific problem it permits researchers to address.

Results obtained with cancer vaccines in several cancer-prone GEM have been reviewed elsewhere [37]. These experiments show that when the time of the experiment is expanded to the significant part of a rodent's natural lifespan, several anti-tumor reaction mechanisms acquire a different and often unforeseen importance [38-39]. A finding common to engrafted tumors and cancer-prone GEM mice is that in both cases, vaccine-induced immune reactivity effectively inhibits early stages of tumor growth, whereas its protective ability vanishes as the tumor progresses.

\section{Into the wind of experimental evidence: vaccines for tumor prevention}

What the numerous immunization-challenge experiments show is how effective the immunity induced against a not-yet-existing tumor is [33]. An almost identical indication comes out from vaccination experiments performed on cancer-prone GEM [26,37]. The experimental rationale for immune prevention is strong because in that setting, immune systems that are neither impaired by tumor-induced and treatmentinduced suppression nor tolerant to TAAs that have been encountered in the absence of correct immunological presentation, are encountered. Five key issues explain why an anti-tumor vaccine should be especially effective: (1) it is administered to a healthy person fully able to mount an immune response; (2) tumor-borne suppressive activities are obviously absent; (3) since no tumor is present, the specific immunity primed by an anti-tumor vaccine should be able to activate adaptive mechanisms towards a superimmune surveillance able to rapidly eradicate a mutated clone; (4) the low proliferation rate of indolent pre-neoplastic lesions cuts down the likelihood of immune-resistant clones selection, while a vaccine-alerted immune response inhibits the lesion prior to complete neoplastic transformation; and (5) precancerous lesions are not yet sheltered from immune attack by the fibroblastic stroma, nor do they display either the tissue organization or significant immunosuppressive abilities of large, proliferating tumor masses $[26,33,37,40]$.

Therefore, why not follow this straightforward experimental indication and consider exploiting vaccination for cancer prevention? Two fundamental obstacles make this endeavor unattractive. The first is that the kind of tumor a person may develop cannot be predicted. Second, it will be exceedingly difficult to get the resources necessary to for a clinical study to 
assess preventive efficacy of a vaccine. Of these two stumbling blocks, the second appears to be the more difficult to overcome. If one could imagine proposing a preventive cancer vaccine to the general population, as is happening now to prevent human papillomavirusinduced tumors, the time necessary to obtain results from a clinical experimentation of this kind and the costs connected would largely overcome the range acceptable for any pharmaceutical enterprise. Moreover, the acceptance of a preventive maneuver is a cold rational choice. A heathy person should accept unknown and long-term risks of autoimmunity in order to fight against a hypothetical cancer risk [41].

However, the issue of vaccines for cancer prevention can be approached step by step. First, slightly distorting the concept of prevention, the efficacy of the vaccine may be tested in patients at risk of recurrence of a conventionally treated cancer. In spite of the massive use of adjuvant cancer treatments, a significant percentage of breast cancer patients will develop tumor recurrences in the four to five years following the end of conventional therapy [42]. Vaccine efficacy could also be tested in persons who display a pre-neoplastic lesion (e.g., oral leukoplakia or basal nevus syndrome) or biochemical correlates (e.g., increased prostate-specific antigen level). Going toward a primary prevention, persons exposed to carcinogens for recreational (tobacco, alcohol), occupational (asbestos, radiations), or medical (cancer survivors) reasons can be considered. [43]. Finally, there are several persons at specific risk of cancer who could benefit from anti-cancer vaccines: individuals carrying genetic alterations that increase the risk of cancer, such as BRCA1/2 families. [44].

The selection of cohorts of people at risk for cancer that may benefit from a preventive anti-cancer vaccine more or less answers also to the first objection on the use of a vaccine to prevent tumors. If the precise tumor a person may develop cannot be predicted, it is expected that epidemiological data combined with genetic analysis will assess the individual risk of cancer as a function of sex, age, family history, genetic makeup, and lifestyle (see the American Cancer Society's Who is at Risk and the Your Disease Risk web sites) [26]. Identification of the gene at risk and its mutated or amplified products would provide a great opportunity to vaccinate against a specific risk of tumor. Gene expression profiles and molecular biology will outline more precisely the probability that a particular TAA will be associated with the tumor for which the person is at risk. The molecular characterization of altered gene products predicted to become TAA in these kinds of tumors will be the first step towards engineering effective tumor-preventive vaccines. By combining this information, researchers can select those persons for whom a specific preventive anti-tumor vaccination might be useful [26].

If a cancer vaccine to prevent tumors is to be considered a rationally acceptable endeavor, one has to define the features of vaccines that can induce and sustain a virtually lifelong immune response as with the vaccines to prevent infectious diseases. The risk that immunological interventions favor the emergence of antigen-loss variants is constantly present in tumor immunology [25]. However, when the time scale of the protection is expanded to many years, this risk is markedly enhanced. In order to minimalize it, a tumorpreventive vaccine should address not a target antigen associated with neoplastic transformation, but instead a target antigen playing a critical role in neoplastic growth and progression. This is an important conceptual distinction among tumor antigens identified on human tumors [19].

Lollini et al. defined oncoantigen tumor targets fulfilling this causal role [26]. When a tumor is driven or requires the action of an oncoantigen, antigen-loss variants are more unlikely. When they may occur, their tumorigenic potential would be markedly impaired. One can also foresee that in the later course of tumor progression, the driving role of the targeted oncoantigen can be taken by different genes whose products, in turn, will offer further oncoantigen targets $[33,37]$.

Tumor cells may maintain a high expression of an oncoantigen and still evade $\mathrm{T}$ cell recognition through the down-modulation of antigen processing machinery and histocompatibility antigens. This is quite a common event, in which tumors sneak through immune control. More than $80 \%$ of all human tumors adopt this strategy to escape the recognition and lysis by cytotoxic $\mathrm{T}$ cells. However, antibody interaction with an oncoantigen expressed on the cell membrane or in the tumor micro-environment is not affected, and antibodies still ensure a functional inhibition of the target oncoantigen. The role of antibodies is multifaceted and depends on the features of targeted oncoantigens. Antibodies not only inhibit the function of a given oncoantigen, but also recruit antibodydependent killer mechanisms and complementdependent reactions. Moreover, forming immunocomplexes, they recruit the host adaptive response and act to some extent as a vaccine [45]. These roles of antibodies in cancer inhibition form a 
new, important, and somewhat unexpected acquisition. Current preclinical and clinical data converge on the relevance of antibodies in antitumor immune responses. Herceptin and other monoclonal antibodies have become efficacious new anti-cancer drugs. Despite these impressive data, most anti-tumor vaccines are still designed to trigger only $\mathrm{T}$ cellmediated immunity, a long-lasting prejudicial heritage stemming from immunization-challenge type of experiments. The comparison of immunoprevention with cancer immunotherapy, which is mainly based on effector $\mathrm{T}$ cells, is again reminiscent of viral immunity, in which cytotoxic $\mathrm{T}$ cells cure infection and antibodies, elicited by primary infection or by vaccines, and provide long-term prevention of reinfection $[33,37]$.

On the basis of the above consideration, Lollini et al. [26,33,37] have outlined three classes of oncoantigens that be targeted by tumor-preventive vaccines.

Class I oncoantigens expressed on the cell surface can be attacked by both antibodies and cell-mediated immunity; they are probably the best target for a preventive vaccine.

Class II oncoantigens are tumor-secreted molecules or molecules in the tumor microenvironment that play essential roles in tumor expansion. These can be targeted by antibodies but not by $\mathrm{T}$ cell-mediated immunity.

Class III oncoantigens are tumor molecules that cannot be reached by antibodies because of their intracellular localization, and thus the membrane expression of their peptides can be targeted only by $\mathrm{T}$ cells.

Numerous data obtained in immunizationchallenge types of experiments as well in different cancer-prone GEM show that vaccine formulations able to elicit antibody and cell-mediated immune responses against oncoantigens efficiently inhibit tumor development $[26,33,37]$. The protection induced in young mice may be boosted and last for the life span of the mice [34]. Vaccines against oncoantigens are especially effective in inhibiting the progression of early stages of cancer, while their efficacy decreases as the tumor progresses. [24,36,38]. Characterization of oncoantigens overexpressed during early stages of chemically induced pre-neoplastic lesions may allow the formulation of vaccines able to trigger a specific and persistent immune memory to be exploited as a fresh strategy for the management of individuals at a high risk for cancer following exposure to a specific carcinogenic agent, for whom no active therapeutic option exists at present [43].

\section{Conclusions}

The long search for tumor antigens has been rewarding since it resulted in the definition of cellular and molecular basis of tumor immunogenicity. Almost every tumor is immunogenic, has to sneak through the meshes of natural immune surveillance [25], and can be a target for vaccine-induced immunity [14]. While the current results observed in patients treated with anti-tumor vaccines are still deceiving, it looks probable that the combination of tumor vaccines with new maneuvers to eliminate tumor-induced immunosuppression [24,32] will soon lead to a new form of cancer management. Whether tumor vaccines targeting oncoantigens will become a new way to prevent cancers that more commonly arise in defined cohorts of the human population is uncertain. However, this possibility discloses a fascinating new scenario for the exploitation of anti-tumor vaccines.

\section{Dedication}

To Piero Cappuccinelli, with admiration and gratitude.

\section{References}

1. Foley EJ (1953) Antigenic properties of methylcholanthrene induced tumors in mice of the strain of origin. Can Res 13: 885-937.

2. Prehn RT, Main JM (1957) Immunity to methyicholanthreneinduced sarcomas. J Nat Cancer Inst 18: 769-778.

3. Klein G, Sjogren HO, Klein E, Hellstrom KE (1960) Demonstration of resistance against methylcholanthreneinduced sarcomas in the primary autochthonous host. Cancer Res 20: 1561-1572.

4. Forni G, Rhim JS, Pickeral S, Shevach EM, Green I (1975) Antigenicity of carcinogen and viral induced sarcomas in inbred and random bred guinea pigs. J Immunol 115: 204210.

5. Dranoff G (2011) Experimental mouse tumour models: what can be learnt about human cancer immunology? Nat Rev Immunol 12: 61-66.

6. Cerottini JC, Brunner KT (1974) Cell-mediated cytotoxicity, allograft rejection, and tumor immunity. Adv Immunol 18: 67-132.

7. Hellström I, Hellström KE (1970) Colony inhibition studies on blocking and non-blocking serum effects on cellular immunity to Moloney sarcomas. Int J Cancer 15: 195-201.

8. Qin Z, Richter G, Schuler T, Ibe S, Cao X, Blankenstein T (1998) B cells inhibit induction of $\mathrm{T}$ cell-dependent tumor immunity. Nat Med 4: 627-629.

9. Hewitt HB (1979) A critical examination of the foundations of immunotherapy for cancer. Clin Radiol 30: 361-369.

10. Hewitt HB, Blake ER, Walder AS (1976) A critique of the evidence for active host defence against cancer, based on personal studies of 27 murine tumours of spontaneous origin. Br J Cancer 33: 241-259. 
11. Herberman RB (1983) Second counterpoint: animal tumor models and their relevance to human tumor immunology. $\mathrm{J}$ Biol Response Mod 2: 217-226.

12. Forni G, Santoni A (1984) Immunogenicity of "nonimmunogenic" tumors. J Biol Response Mod. 3:12831.13. Boon T,Van Pel A (1978) Teratocarcinoma cell variants rejected by syngeneic mice: protection of mice immunized with these variants against other variants and against the original malignant cell line. Proc Natl Acad Sci U S A 75: 1519-1523.

13. Boon T, Van Pel A (1978) Teratocarcinoma cell variants rejected by syngeneic mice: protection of mice immunized with these variants against other variants and against the original malignant cell line. Proc Natl Acad Sci U S A 75: 1519-1523.

14. Boon T (1983) Antigenic tumor cell variants obtained with mutagens. Adv Cancer Res 39: 121-151.

15. Shevach EM, Rosenthal AS (1973) Function of macrophages in antigen recognition by guinea pig $\mathrm{T}$ lymphocytes. II. Role of the macrophage in the regulation of genetic control of the immune response. J Exp Med 138: 1213-1229.

16. Traversari $C$, van der Bruggen $P$, Luescher IF, Lurquin $C$, Chomez P, Van Pel A, De Plaen E, Amar-Costesec A, Boon T (1992) A nonapeptide encoded by human gene MAGE-1 is recognized on HLA-A1 by cytolytic T lymphocytes directed against tumor antigen MZ2-E. J Exp Med 176: 1453-1457.

17. Coulie PG, Van den Eynde BJ, van der Bruggen P, Boon T (2014) Tumour antigens recognized by T lymphocytes: at the core of cancer immunotherapy. Nat Rev Cancer 14: 135-146.

18. Moretta L, Montaldo E, Vacca P, Del Zotto G, Moretta F, Merli P, Locatelli F, Mingari MC (2014) Human natural killer cells: origin, receptors, function, and clinical applications. Int Arch Allergy Immunol 164: 253-264.

19. Cheeever MA, Hastings BM, Viner JL, Allison JP, Hecht TT, Weiner LM, Ferris AS, Mellman I, Matrisian LM, Finn OJ, Prindiville SA (2009) The Prioritization of Cancer Antigens: A National Cancer Institute Pilot Project for the Acceleration of Translational Research. Clin Cancer Res 15: 5323-5337.

20. Calogero RA, Quaglino E, Saviozzi S, Forni G, Cavallo F (2008) Oncoantigens as anti-tumor vaccination targets: The chance of a lucky strike? Cancer Immunol Immunother 57: 1685-1694.

21. Iezzi M, Calogero RA, Spadaro M, Musiani M, Forni G, Cavallo F (2012) BALB-neuT Female Mice as a Dynamic Model of Mammary in Translational Animal Models. In Wang X, editor. Drug Discovery and Development. Beijing: Bentham Science Publishers. 139-166.

22. Harzstark AL, Small EJ (2007) Immunotherapy for prostate cancer using antigen-loaded antigen-presenting cells: APC8015 (Provenge). Expert Opin Biol Ther 7: 1275-1280.

23. Provinciali M, Smorlesi A, Donnini A, Bartozzi B, Amici A (2003) Low effectiveness of DNA vaccination against HER2/neu in ageing. Vaccine 21: 843-848.

24. Bolli E, Quaglino E, Arigoni M, Lollini PL, Calogero R, Forni G, Cavallo F (2011) Oncoantigens for an immune prevention of cancer. Am J Cancer Res 1: 255-264.

25. Dunn GP, Old LJ, Schreiber RD (2004) The Three Es of Cancer Immunoediting. Annu Rev Immunol 22: 329-360.

26. Lollini PL, Cavallo F, Nanni P, Forni G (2006) Vaccines for cancer prevention. Nature Rev Cancer 6: 204-216.

27. Couzin-Frankel J (2010) Immune therapy steps up the attack Science 330: 440-443.
28. Rolla S, Ria F, Occhipinti S, Di SG, Iezzi M, Spadaro M, Nicolo C, Ambrosino E, Merighi IF, Musiani P, Forni G, Cavallo F (2010) Erbb2 DNA vaccine combined with regulatory $\mathrm{T}$ cell deletion enhances antibody response and reveals latent low avidity $\mathrm{T}$ cells: potential and limits of its therapeutic efficacy. J Immunol 184: 6124-6132.

29. Piconese S, Valzasina B, Colombo MP (2008) OX40 triggering blocks suppression by regulatory $\mathrm{T}$ cells and facilitates tumor rejection. J Exp Med 205: 825-839.

30. Ostrand-Rosenberg S (2010) Myeloid-derived suppressor cells: more mechanisms for inhibiting antitumor immunity. Cancer Immunol Immunother 59: 1593-1600.

31. Ugel S, Delpozzo F, Desantis G, Papalini F, Simonato F, Sonda N, Zilio S, Bronte V (2009) Therapeutic targeting of myeloid derived suppressor cells. Curr Opin Pharmacol 9: 470-481.

32. Wolchok JD, Chan TA (2014) Antitumour immunity gets a boost. Nature 515: 496-497.

33. Cavallo F, De Giovanni C, Nanni P, Forni G, Lollini PL (2011) 2011: The immune hallmarks of cancer. Cancer Immunol Immunother 60: 319-326.

34. Quaglino E, Rovero S, Cavallo F, Musiani P, Amici A, Nicoletti G, Nanni P, Forni G (2002) Immunological prevention of spontaneous tumors: a new prospect? Immunol Lett 80: 75-79.

35. Cavallo F, Offringa R, van der Burg SH, Forni G, Melief CJ (2006) Vaccination for treatment and prevention of cancer in animal models. Adv Immunol 90: 175-213.

36. Quaglino E, Mastini C, Amici A, Marchini C, Iezzi M, Lanzardo S, De Giovanni C, Montani M, Lollini PL, Masucci G, Forni G, Cavallo F (2010) A better immune reaction to Erbb-2 tumors is elicited in mice by DNA vaccines encoding rat/human chimeric proteins. Cancer Res 70: 2604-2612.

37. Lollini PL, Nicoletti G, Landuzzi L, Cavallo F, Forni G, De Giovanni C, Nanni P (2011) Vaccines and Other Immunological Approaches for Cancer Immunoprevention. Current Drug Targets 12: 1957-1973.

38. Nanni P, Landuzzi L, Nicoletti G, De Giovanni C, Rossi I, Croci S, Astolfi A, Iezzi M, Di Carlo E, Musiani P, Forni G, Lollini P-L (2004), Immunoprevention of Mammary Carcinoma in HER-2/neu Transgenic Mice is $\gamma$-Interferon and B Cell Dependent. J Immunol 173: 2288-2296.

39. Quaglino E, Rolla S, Iezzi M, Spadaro M, Musiani P, De Giovanni C, Lollini PL, Lanzardo S, Forni G, Sanges R, Crispi S, De Luca P, Calogero R, Cavallo F (2004) Concordant morphologic and gene expression data show that a vaccine halts HER-2/neu preneoplastic lesions. J Clin Invest 113: 709-717

40. Forni G, Curcio C, Spadaro M, Iliffe J, Quaglino E, Di Carlo E, Musiani P, Lollini P (2003) Immunization in tumor prevention. Intern Immunopharmacol 3: 1151-1158.

41. Forni G, Lollini PL, Musiani P, Colombo MP (2000) Immunoprevention of cancer: is the time ripe? Cancer Res 60: 2571-2575.

42. Becker S, Solomayer E, Becker-Pergola G, Wallwiener D, Fehm T (2007) Primary systemic therapy does not eradicate disseminated tumor cells in breast cancer patients. Breast Cancer Res Treat 106: 239-243.

43. Berta GN, Sprio AE, Iezzi M, Spadaro M, Cappia S, Salamone P, Di Scipio F, Mognetti B, Papotti M, Musiani P, Forni G, Cavallo F (2011) A DNA vaccine against ERBB2 impairs chemical carcinogenesis in random-bred hamsters. Cancer Prev Res 4: 994-1001. 
44. McCann GA, Eisenhauer EL (2015) Hereditary cancer syndromes with high risk of endometrial and ovarian cancer: surgical options for personalized care J Surg Oncol 111: 118124.

45. Park S, Jiang Z, Mortenson ED, Deng L, Radkevich-Brown O,Yang X, Sattar H, Wang Y, Brown NK, Greene M, Liu Y, Tang J, Wang S, Fu YX (2010) The therapeutic effect of antiHER2/neu antibody depends on both innate and adaptive immunity. Cancer Cell 18: 160-170.

\section{Corresponding author}

Guido Forni

Accademia Nazionale dei Lincei

Via della Lungara 10

00165 Roma, Italy

Phone: + 00393474089236

Email: frngdu@gmail.com

Conflict of interests: No conflict of interests is declared. 